\title{
Fusion and the cosmos
}

\author{
H.Wilhelmsson \\ Department of Electromagnetics, Chalmers University of Technology \\ and EURATOM - VR Association, \\ SE-41296 Göteborg, Sweden
}

Received March 16, 2004

\begin{abstract}
In the following investigation we pay special attention to the role of selforganization in fusion plasma physics and in the cosmos. We present a new approach to the expansion of the universe. Formally the technique developed relies on our experience from treating hot fusion plasmas. We account for the possibility that the universe, as it seems, could have a finite life-time (even if it is counted in billions of years), and combine this assumption with the experimental observation that the velocity of separation of distant galaxies is proportional to the distance between the galaxies (the Hubble law). By analysis of a NL PDE (nonlinear partial differential equation) we succed in proving that the crucial value of an exponent has a simple linear relationship with the Hubble constant. It is recognized that the scale-length that we use as a measure of the expansion is equivalent to the Einstein radius of curvature. The final results suggest that the Hubble law should be extended by a factor, which could have an explosive tendency of growth in time (open universe), or a decaying character (closed universe). The possibility of reversed expansion or an oscillating universe "cosmic pendulum" is also discussed.
\end{abstract}

Key words: self-organization in the cosmos, Hubble law, oscillating universe

PACS: 98.80.Es, $91.01 \mathrm{Qm}$

\section{Introduction}

Space is full of fields and particles. The fields could be gravitational, magnetic, electric etc ... and the particles: protons, neutrons, electrons, neutrinos etc. These may be more or less coupled to each other, depending on the situation (position and time). There are even smaller entities: quarks, building up the nuclei (protons and neutrons) and perhaps others still not discovered. The particles can be grouped together forming more complex systems like atoms, molecules or plasmas (free ions and electrons at high temperatures). The motion of such systems depends on their inertia as well on the presence of fields [1-4]. The way in which the grouping occurs can be described as self-organization and may lead to self-formation. The fields as 
well as the particles participate in such formations.

The self-formation of e.g. a fusion plasma may result in bell-shaped stuctrures, exhibiting the impressive peculiarity of so-called profile self-consistency, a tendency to retain optimal radial distribution profiles of plasma and temperature [5-10]. Such profiles resist any attempts to modify them, e.g. by a change of external power deposition profile [7-14]. These properties are of great interest when considering the formation of equilibria of fusion plasmas as well as the stability of such equilibria, in fusion laboratory experiments and in the cosmos [20-24].

The process of self-formation may also lead to filamentation and striation structures of plasmas, in particular in the cosmos, as well as whirls and vortices [2-7], e.g. in the Aurora Borealis [25], in magnetospheric shock-wave and tail formation, but also as magnetic island structures affecting fusion plasma transport as well as current profile control in fusion plasmas [14].

In figures 1 and 2 are exposed the results of magnificent self-organization in Nature and in the Cosmos. Note that more than four centuries passed in between the two events realized by Leonardo and Vincent, outstanding artists of the last millenium.

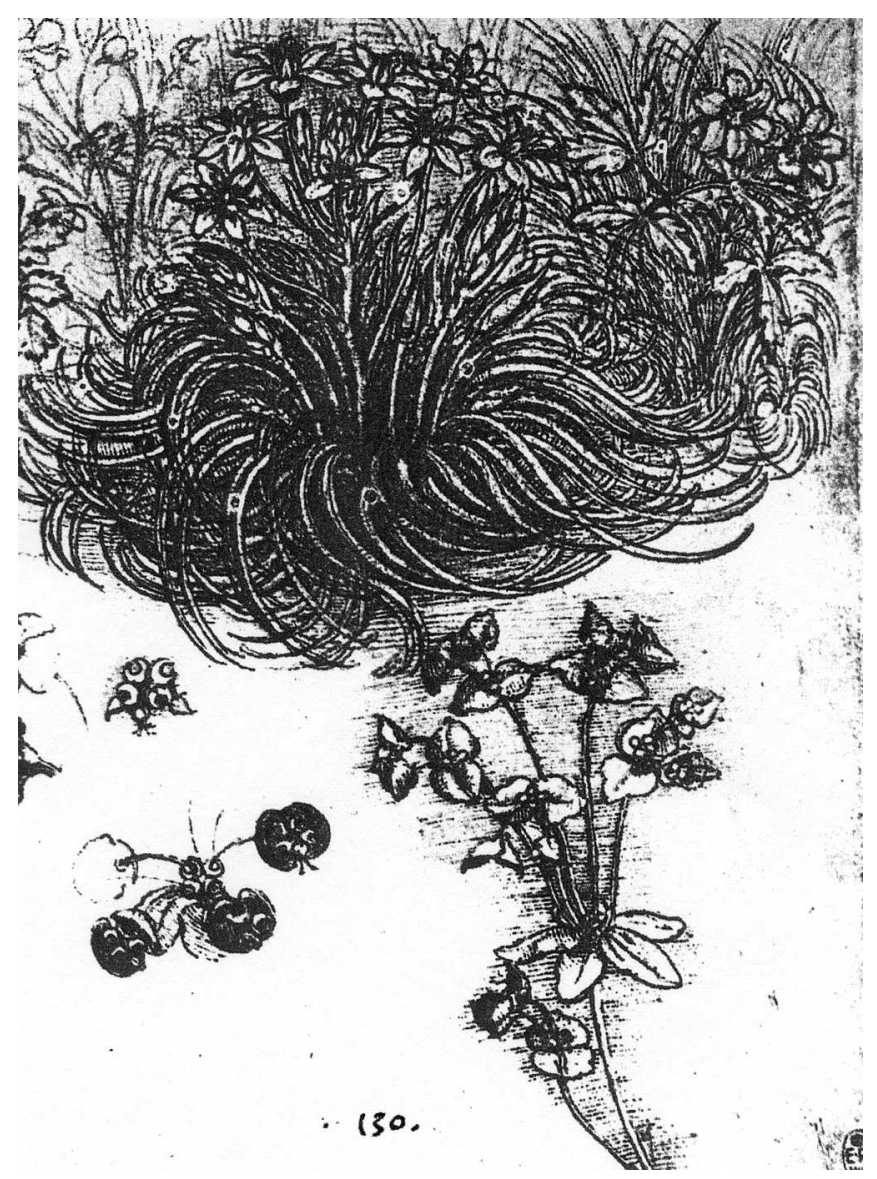

Figure 1. "Bethleém's star". Drawing by Leonardo da Vinci, propably around 1480 (Windsor, Royal Library). 


\section{Self-organization}

Self-organization occurs in all areas of science, in our daily life, in atomic, molecular, nuclear and plasma processes, in the formation of stars and galaxies as well as in the evolution of the universe as a whole [1-3].

In the interstellar medium self-organization could start from small local density perturbations which develop due to gravitational contraction into density clouds and by subsequent ionization and nuclear fusion into star formation. Self-formation in a star occurs under the influence of gravitational forces and the thermonuclear pressure. Similarly, one tries to form in the fusion laboratories, configurations for which, by means of magnetic or inertial confinement, fusion energy balances losses by diffusion and radiation[4-14]. As a consequence of the powers involved, the way to self-formation of an equilibrium state by self-organization is governed by nonlinear coupling processes in between the participating variables. In particular the diffusion is a typically nonlinear process that influences the dynamical behaviour and the equations used to describe the evolution.

These equations may be cast into a nonlinear partial differential form to describe the laboratory fusion plasmas and will be developed here in a similar form for the use of describing the expansion of the universe [3].

Several approaches might, in fact, be used to describe the evolution. The most general way would be to make detailed investigations of all the elementary nonlinear mechanisms which lead to self-organization and try to combine all the result into one integrated picture of complex behaviour [10]. Another way would be to use simple physical arguments based on numerical models.

A complete description of all the simultaneous detailed features is naturally outside the scope of any attempt. In the theory describing the combined effects of gravitation, quantum mechanics and particle physics ambitious scientists started to talk about the equation of everything in order to obtain a unified theory of nature to be compared with experimental results. This would still have a long way to go and the solution to such an equation could hardly provide, as it seems, comprehensive results, all elements taken into account.

In particular the comparison with experimental results would still remain even if it is said that the developments in modern cosmology give some hope... To us it seems convenient to make use of a description in terms of "concentrated variables" i.e. amplitudes and profile parameters [4]. In the present investigation the deduction is done in two steps, considering cases, where the shape of the solution, (i) does not change, or (ii) the shape does permit a proper change in the process of evolution.

In the presentation we do not pay specific attention to quantum effects that may prevail for extreme densities of nuclear condensed matter in supernovas, white dwarfs or black holes where they may be responsible for radiative effects. Such phenomena may however be said to be accounted for by the values of the "concentrated variables" and parameters characterizing the dynamics of the system that we describe.

In the NL PDE (nonlinear partial differential equation) the nonlinearity appears 
explicitly in terms of a power dependence in the diffusion coeffcient on the amplitude (density or temperature).

An interesting result of the investigation is a simple expression which relates the NL exponent to the Hubble constant. Our NL PDE thus provides a dynamic equation for the universe, which might even be extended to account for the influence of sources similarly to the case of a hot fusion plasma.

It seems that the convensional form of the Hubble law might have to be modified by a certain factor introduced by a change in form of the universe in the process of expansion. This seems indeed to be rather natural for an evolution approaching a crunch state.

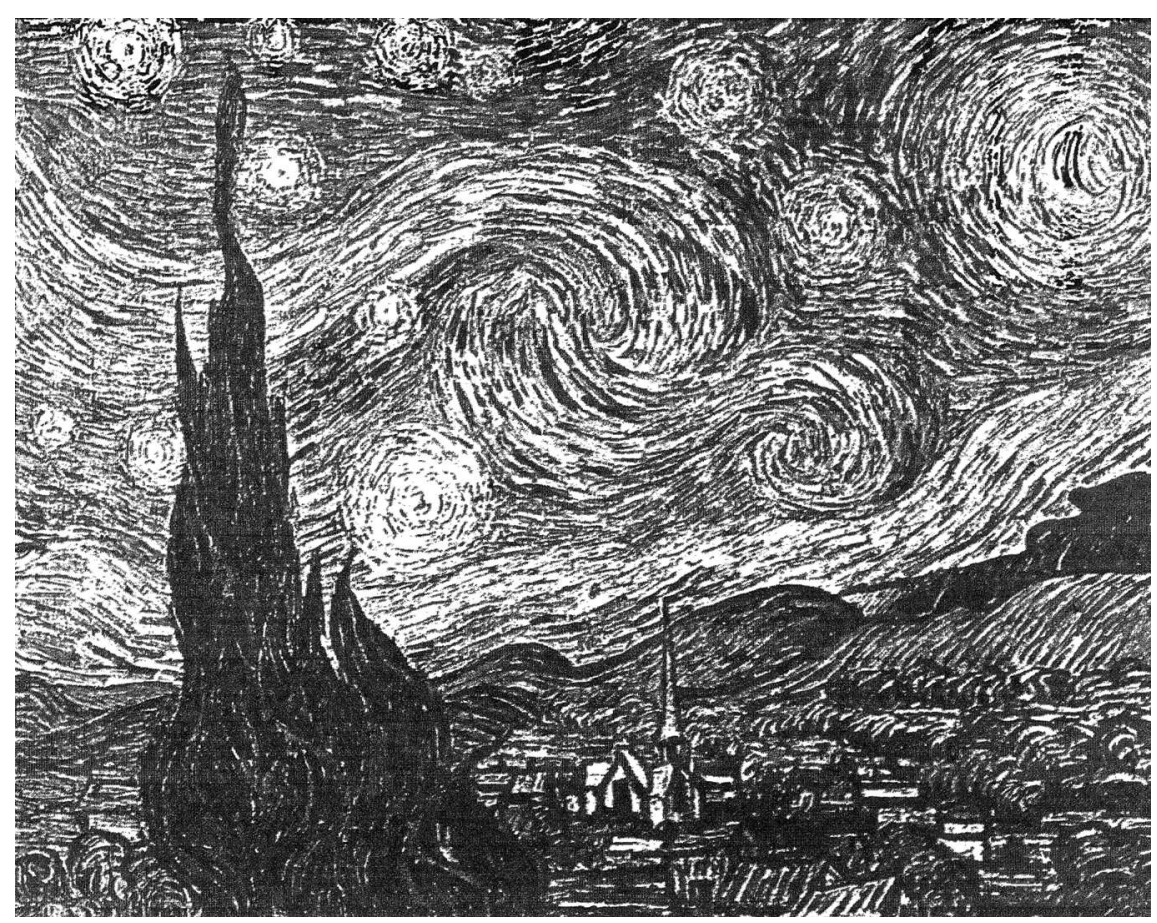

Figure 2. "Starry Night": 1889 oil painting by Vincent Van Gogh (Courtesy of Museum of Modern Art, New York).

\section{Universal expansion and an extended Hubble law}

\subsection{Basic NL PDE and solutions}

It would be temping to try a nonlinear diffusion type of description for the expanding universe similar to the one successfully used for particle transport and temperature conductivity in fusion plasma physics. A formal study could be done in terms of a quantity $u$, representing the local density variation in space and time, $u=n(x, t)$, where

$$
u=A\left(1-\frac{x^{2}}{L^{2}}+\eta \frac{x^{4}}{L^{4}}\right)
$$


with $A=A(t), L=L(t)$ time-dependent quantities, representing amplitude, scalelenght, and where $\eta=\eta(t)$ is a time-dependent form factor. As a rule it is sufficient to keep terms only up to the fourth order in $x$.

An expression for the quantity of flow could then be written

$$
F=a u^{\alpha} \frac{\partial u(x, t)}{\partial x}
$$

where $a$ and $\alpha$ are constants.

The form of the nonlinear partial differential equation (NL PDE) governing the universal expansion can accordingly be expressed

$$
\frac{\partial u}{\partial x}=a x^{-\gamma} \frac{\partial}{\partial x}\left(x^{\gamma} u^{\alpha} \frac{\partial u}{\partial x}\right)
$$

where $\gamma$ is related to the dimension $d$ as $\gamma=d-1$.

Introducing the expression (1) for $u$ into equation (2) and matching the increasing orders of $x^{2}$-terms $\left(x^{0}, x^{2}, x^{4}\right)$ one obtains the following set of ordinary coupled nonlinear first order differential equations for $A, L^{2}$ and $\eta$, namely

$$
\begin{aligned}
\frac{\mathrm{d} A}{\mathrm{~d} t} & =-2 a(\gamma+l) \frac{A^{\alpha+l}}{L^{2}} \\
\frac{\mathrm{d} L^{2}}{\mathrm{~d} t} & =2 a \Gamma A^{\alpha} \\
\frac{\mathrm{d} \eta}{\mathrm{d} t} & =a \Lambda \frac{A^{\alpha}}{L^{2}}
\end{aligned}
$$

If one assumes that the universe expands without large-scale deformation one has $(\mathrm{d} \eta) / \mathrm{d} t=0$, and $\Lambda=0$ in equation (6). Solutions to equations (4) and (5) can be expressed in the form

$$
\begin{aligned}
\frac{A}{A_{0}} & =\left(1+\frac{t}{t^{\prime}}\right)^{\mu}, \\
\left(\frac{L}{L_{0}}\right)^{2} & =\left(1+\frac{t}{t^{\prime}}\right)^{\nu},
\end{aligned}
$$

where for a spherically symmetric universe $(d=3, \gamma=2)$

$$
\begin{aligned}
& \mu=-3(3 \alpha+\Gamma)^{-l} \\
& \nu=\Gamma(3 \alpha+\Gamma)^{-l}
\end{aligned}
$$

and

$$
\Gamma=5(\alpha+2 \eta)-3
$$

It should be noted that the quantity $L(t)$ may be interpreted as a scale-length of the distribution of matter in space, or as the forward base-point of the distribution (1) with $u=0$ and $\eta=0$, describing the evolution of the temporal outer limit of the universe. It can, however, also be recognized as an equivalent to the Einstein radius 
of curvature in space (see [3]). It is important to emphasize, in this connection, that the particular positions of stars and galaxies etc, i.e. their specific $x$-coordinates, are now relaxed in the description, which accounts only for the expansion of a smooth distribution, as a celestial fluid, averaged everywhere over a large number of objects.

The characteristic time $t^{\prime}$ in the relations (7-8) can be expressed as

$$
t^{\prime}=\frac{L_{0}^{2}}{2 a A_{0}(3 \alpha+\Gamma)}
$$

One notices from equations (7) and (8) that $A$ and $L^{2}$ obey the following constant of motion relation, namely

$$
\left(\frac{A}{A_{0}}\right)^{\Gamma / 3}\left(\frac{L}{L_{0}}\right)^{2}=1
$$

where in equations (12) and (13) $A_{0}$ and $L_{0}$ denote initial conditions, related if one so wishes to an instant of the early phases of the Big Bang $\left(t=0, L=L_{0}\right)$.

It follows from equation (5) and the solutions (7) and (8) that one may express

$$
V=\frac{\mathrm{d} L}{\mathrm{~d} t}=\left(a \Gamma \frac{A_{0}^{\alpha}}{L_{0}^{2}}\right) L\left(\frac{L^{2}}{L_{0}^{2}}\right)^{\alpha \mu / \nu-1}
$$

where

$$
\alpha \mu / \nu-1=-\frac{(3 \alpha+\Gamma)}{\Gamma}
$$

which can easily be compared with the Hubble law

$$
V=\frac{\mathrm{d} L}{\mathrm{~d} t}=H L
$$

i.e.

$$
L=\frac{L_{0}}{\exp (H t)},
$$

where $H$ denotes the Hubble constant. One notices that one has to impose the condition $\alpha \mu / \nu-1=0$ or $(3 \alpha+\Gamma) / \Gamma=0$ in equation (14) to obtain the characteristic linear dependence of the expansion velocity $V$ on the distance $L$ expressed by the Hubble relation (15).

As a result one obtains from the expressions (9)-(11) the remarkably simple relation

$$
(3 \alpha+\Gamma)=0
$$

and from the equations (14)-(16)

$$
\alpha=-\frac{\Gamma}{3}=-\frac{H L_{0}^{2}}{3 a A_{0}^{\alpha}},
$$

which settles the value of $\alpha$ to be $\alpha=3 / 8=0.375$ and $\Gamma=-9 / 8=-1.125$ for $\mathrm{d} \eta / \mathrm{d} t=0$ with $\Lambda=0$ and $\eta=0$, using the expressions (11) and (16).

From the expression (17) we notice the interesting linear relationships between $\alpha$ and $H$ and also between $\alpha$ and $\Gamma$, as well as the dependence of $\alpha$ on the initial values of $L$ and $A$, which agrees with the dimentional form of the equation (3). 


\subsection{Extended formulation}

The relation (16) corresponds, in fact, to a solution, for which the parameters $\eta$ and $\nu$ as well as the characteristic time $t^{\prime}$ reach unlimited values, according to the expressions (9), (10) and (12).

From the formulas (7)-(12), and considering $3 \alpha+\Gamma$ approaching zero, the values of $A(t)$ and $L(t)$ can, however, be obtained for small values of $t(H t \ll 1)$, namely

$$
\frac{A}{A_{0}}=l-6 \Gamma^{-l} H t, \quad(\Gamma<0), \quad \frac{L}{L_{0}}=1+H t
$$

as well as from equation (14)

$$
V=\frac{\mathrm{d} L}{\mathrm{~d} t}=H L_{0}(1+H t)
$$

To obtain a consistant and physically meaningful description by means of the equations (4)-(6) with (7)-(12), and to use the proper direction of time, related to the sign in the Hubble law (15), one has to avoid the singularity by an amount $\varepsilon$ such that

$$
3 \alpha+\Gamma=-\varepsilon
$$

or

$$
\alpha \mu / \nu-1=\varepsilon / \Gamma, \quad \mu=3 / \varepsilon, \quad \nu=-\Gamma / \varepsilon,
$$

where $\varepsilon<0$ or $\varepsilon>0$, corresponding to an open or closed universe, respectively. One notices from the expression (12) with the relation (18) and $T=-t^{\prime}>0$, $\varepsilon<0, \Gamma<0, a<0$ that

$$
\varepsilon=\frac{L_{0}^{2}}{2 a A_{0}^{\alpha} T}=\frac{\Gamma}{2 H T}
$$

and

$$
\frac{L}{L_{0}}=\left(1-\frac{t}{T}\right)^{-H T}, \quad(t<T)
$$

or

$$
V=\frac{\mathrm{d} L}{\mathrm{~d} t}=H L\left(l-\frac{t}{T}\right)^{-l}=H L\left(\frac{L}{L_{0}}\right)^{1 / H T},
$$

where the Hubble constant, from the relations (14) and (15) is

$$
H=a \Gamma \frac{A_{0}^{\alpha}}{L_{0}^{2}}, \quad(a<0, \Gamma<0)
$$

and

$$
T=\frac{\Gamma}{2 H \varepsilon}, \quad(\Gamma<0, \varepsilon<0) .
$$

As a result one notices that the expansion velocity $V$ in the equation (21) includes an enhancement factor of explosive character, namely $(1-t / T)^{-1}$ with respect to the conventional Hubble law. For finite $T$, i.e. non-zero $\varepsilon$ the critical time $t=T$, is finite whereas for $\varepsilon=0, T$ becomes infinite. 
It is interesting to notice that the scale-lenght $L(t)$ here used (1), (4)-(6), (8), (13)-(15), (21) turns out to be equivalent to the Einstein radius of curvature $R(t)$, (see [3]). The restriction (16), i.e. $3 \alpha+\Gamma=0$ is not consistent with the requirement that $T$ could be finite since from the expression (12) $T$ would be proportional to $(3 \alpha+\Gamma)^{-1}$ and therefore infinite. The origin of this discrepancy is that the classical Hubble law has the precise form $V=H L$. Slight changes in the form-parameters $\eta$, etc. would not essentially change this discrepancy, but only introduce small relative changes in $\alpha$ versus $\Gamma$, which would seem non-consistent with shape-preservation, i.e. $\mathrm{d} \eta / \mathrm{d} t=0$, and may be considered more a question of informatics. The conclusion would be that one has to make an extension of the Hubble law according to the relation (21), corresponding to an open universe (or for $\varepsilon>0$ to a closed universe), or to change the form of the NL PDE equation, which does not seem attractive!

\section{Conclusions and discussion}

It seems that the NL PDE has support from many corners of science, and besides is more general and possibly more far-reaching than the specific Hubble form. Further detailed measurements may provide an answer or at least an indication to this subtle but principally important point. What would finally be the value of the coefficient $\alpha$ and how would it be related to the time T? From the equations (11), (18), (19) the simple form for $\alpha$ in the NL PDE $(\gamma=2 ; d=3)$ becomes

$$
\alpha=-\frac{H L_{0}^{2}}{3 a A_{0}^{\alpha}}\left(1+\frac{3}{16 H T}\right) .
$$

where the second term in the parenthesis accounts for the influence of a finite value of $T$, which also influences $\Gamma$, namely

$$
\Gamma=\frac{H L_{0}^{2}}{a A_{0}^{\alpha}}\left(1+\frac{5}{16 H T}\right)^{-1}, \quad H T>1
$$

with

$$
\frac{H L_{0}^{2}}{a A_{0}^{\alpha}}=-\frac{9}{8}
$$

from the relations (11) and (16) for $(1 /[H T])=0, \varepsilon=0$.

For an infinitely large value of $T$ one recovers in (24) the value $\alpha=3 / 8$ from equation (17). The relations (24) and (26) are particularly interesting since they link together $\alpha$ with the Hubble constant $H$, with $T$, and with the initial values of $L_{0}$ and $A_{0}$ as well as with the linear diffusion coefficient $a$, see expressions (2), (3). The relations $(24),(25)$ refer to an open universe $(\varepsilon<0)$. For a closed universe the signs in the parentheses of (24), (25) should be changed.

One might imagine to use our formulation even to model how a turning, i.e. a reversal of the expansion, could be described. Before reaching a critical domain, or a crunch, we could assume that the universe did not change in shape with time, which meant that $\mathrm{d} \eta / \mathrm{d} t=0$, with $\eta=0$, corresponding to $\Lambda=0$ in the expression 
(6) and $\Gamma=-9 / 8, \alpha=3 / 8$ from the relations (11) and (16). Now approaching a crash this cannot possibly be true. The conditions have to be changed. Near the "turning" one would expect not only $\varepsilon=0$ in (18) but also $\Gamma=0$ and $\alpha=0$ from the relations (14)-(16), which happens for a particular value $\eta=0.3$ in (11).

The turning would then be described by a transition from $\mathrm{d} \eta / \mathrm{d} t=0$ with $\eta=0$ $(\Gamma=-9 / 8, \alpha=5 / 3, \varepsilon$ different from 0$)$ to $\mathrm{d} \eta / \mathrm{d} t$ different from 0 with $\eta=0.3$ $(\Gamma=0, \alpha=0, \varepsilon=0)$, and with a reversal in time to return to the previous shape and distribution (backwards in time!), not a detailed one of course, but an average one. That means to return by contraction and compression, and by heating up the matter to the state of the original Big Bang from the intermediate Big Crunch!

Enormous amounts of matter may be concentrated, in both or either of these limits, forming a hot plasma, ejected particles and radiation of X-rays, neutrinos etc. Perhaps, it would mark the beginning of a new phase in the motion of the "cosmic pendulum", an oscillating universe, where the masses of ejected dust and crashed matter would again form new galaxies and stars to be thrown out in space, with enormous forces to later on contract again into a small total volume and form the beginning of the next universe and so on. But that is another story!

One may, however, consider the Hubble expansion as a specific manifestation of what could be considered, in a more general sense, nonlinear cosmodynamics (NL CD). The above analysis may be seen as an attempt to approach such a description by means of a certain form of nonlinear partial differential equations (NL PDE). It extends ideas based on a recent paper by the author [2], relating fusion plasma physics and gravitation.

\section{References}

1. Klimontovich Yu.L., Wilhelmsson H., Yakimenko I.P., Zagorodny A.G. Statistical theory of plasma-molecular systems. Physics Reports, vol. 175, p. 264-401, 1989; Moscow University Press, 223p. (in Russian), cf. also [15-19].

2. Wilhelmsson H. Fusion, a voyage through the plasma universe. Bristol, Philadelphia: IOP Publ, 2000.

3. Wilhelmsson H. Gravitational contraction and fusion plasma burn. Universal Expansion and the Hubble Law. Physica Scripta, vol. 66, p. 395, 2002.

4. Wilhelmsson H., Lazzaro E. Reaction-diffusion problems in the physics of hot Plasmas. Bristol and Philadelphia: IOP Publ, 2001.

5. Coppi B. Nonclassical transport and the "principle of profile consistency". Comments on Plasma Phys. Contr. Fusion, vol. 5, p. 261, 1980.

6. Coppi B. Profile consistency: global and nonlinear transport // Phys. Lett. A, 1988, vol. 128, p. 183.

7. Wilhelmsson H. Self-formation and evolution of singletons // Int. J. Quantum Chem., 1989, vol. 35, p. 887.

8. Wilhelmsson H. Diffusion, creation and decay processes in plasma dynamics: evolution towards equilibria and the role of bifurcated states // Nuclear Physics A, 1990, vol. 518 , p. 84 . 
9. Wilhelmsson H. Evolution and self-formation in some nonlinear systems // Bulletin de la Classe des Sciences, Academie Royale de Belgique, No. 6, vol. 1,10-11, p. 269-287 (invited talk)/

10. Kadomtsev B.B. Tokamak plasma: a complex physical system. Bristol and Philadelphia: IOP Publ, 1992.

11. Wilhelmsson H. Global dynamics of thermonuclear fusion plasmas: self-consistent treatment of diffusion-reaction equations, (selected papers A1-A26 1987-1994), CTHIEFT/PP-1994-01 ISSN 0281-1308, 1994.

12. Wilhelmsson H., Lazzaro E., Cirant S. Sensitivity of fusion plasma temperature profiles to localized and distributed heat sources // Physica Scripta, 1996, vol. 54, p. 385.

13. Lazzaro E., Wilhelmsson H. Fast heat-pulse propagation in hot plasmas // Phys. of Plasmas, 1998, vol. 8, p. 2830.

14. Itoh K., Itoh S.I., Fukuyama A. Transport and structural formation in plasmas. Bristol and Philadelphia: IOP Publ., 1999.

15. Wilhelmsson $\mathrm{H}$. Resonant interaction between an active molecular medium and a free-electron laser // Physica Scripta, 1980, vol. 22, p. 501.

16. Stenflo L., Wilhelmsson H. Radiation from a relativistic electron bean in a molecular medium due to parametric pumping by a strong electomagnetic wave // Phys. Rev. A, 1981, vol. 24, p. 1115.

17. Wilhelmsson H., Gustavsson H.-G., Stenflo L. Generation of high power, very coherent radiation of a free electron laser with a molecular (or ionic) medium // Physica Scripta, 1983, vol. 28, p. 374 .

18. Wilhelmsson H. Double-beam free electron laser // Physica Scripta, 1991, vol. 44, p. 184.

19. Wilhelmsson H., Etlicher B., Cairns R.A., le Roux M.-N. Evolution of temperatures profiles in a fusion reactor plasma // Physica Scripta, 1992, vol. 45, p. 184.

20. Wilhelmsson H., le Roux M.-N. Equilibria, scaling laws and density in alpha particle heated fusion plasma // Physica Scripta, 1992, vol. 46, p. 375.

21. le Roux M.-N., Weiland, Wilhelmsson H. Simulation of a coupled dynamic system of temperature and density in a fusion plasma // Physica Scripta, 1992, vol. 46, p. 457.

22. Wilhelmsson H., le Roux M.-N. Self-consistent treatment of transport in tokamak plasmas // Physica Scripta, 1993, vol. 48, p. 735.

23. Wilhelmsson H., Gustavsson H.-G. Dynamic response treatment of a burning fusion plasma // Physica Scripta, 1994, vol. 49, p. 715.

24. Lundin R., Marklund G. Plasma vortex structures and the evolution of the solar system-the legacy of Hannes Alfvén // Physica Scripta, 1995, vol. T60, p. 198. 


\section{Термоядерний синтез і космос}

\section{Г.Вільгельмсон}

Кафедра електромагнетизму технологічного університету Халмера і асоціація ЄВРОАТОМ,

SE-41296 Гетеборг, Швеція

Отримано 16 березня 2004 р.

Особлива увага в даному дослідженні приділяється ролі самоорганізації у фізиці термоядерної плазми і у Всесвіті. Представлено новий підхід до проблеми розширення Всесвіту. Формально розвинений підхід ґрунтується на досвіді, отриманому при вивченні гарячої термоядерної плазми. Ми враховуємо можливість того, що Всесвіт, як видається, може мати скінчений час життя (навіть якщо рахунок йде на мільярди років), та поєднуємо це припущення з експериментальним фактом, що швидкість розбігання віддалених галактик пропорційна до віддалі між ними (закон Габбла). На основі аналізу неоднорідного диференціального рівняння у частинних похідних нам вдалося довести, що критичне значення показника пов'язане зі сталою Габбла простим лінійним співвідношенням. Виявлено, що масштаб, який ми використовуємо як міру розширення Всесвіту, є еквівалентним до ейнштейнівського радіусу кривизни. 3 кінцевих результатів випливає, що закон Габбла слід доповнити множником, який може мати вибухоподібну тенденцію росту з часом (відкритий Всесвіт), або спадний характер (замкнений Всесвіт). Обговорюється можливість оборотнього розширення або осцилюючого Всесвіту (космічний маятник).

Ключові слова: самоорганізація у Всесвіті, закон Хаббла, осцилюючий Всесвіт

PACS: $98.80 . E s, 91.01 \mathrm{Qm}$ 
